Santé mentale au Québec

Plan d'action québécois en santé mentale : contextes de mise en oeuvre et éléments d'impact sur l'organisation des services de première ligne et les modes de collaboration

Mental Health Action Plan: Contextual analysis and elements impacting on

Plan de acción quebequense en salud mental: contextos de la implementación y elementos de impacto en la organización de los servicios de primer nivel y las formas de colaboración

Plano de ação quebequense em saúde mental: contextos de aplicação e elementos de impacto na organização dos serviços primários e os modos de colaboração

Catherine Vallée, Léo-Roch Poirier, Denise Aubé, Louise Fournier, Malijaï Caulet, Pasquale

Roberge et Lily Lessard

Volume 34, numéro 1, printemps 2009

Santé mentale en première ligne

URI : https://id.erudit.org/iderudit/029758ar

DOI : https://doi.org/10.7202/029758ar

Aller au sommaire du numéro

Éditeur(s)

Revue Santé mentale au Québec

ISSN

0383-6320 (imprimé)

1708-3923 (numérique)

Découvrir la revue

Citer cet article

Vallée, C., Poirier, L.-R., Aubé, D., Fournier, L., Caulet, M., Roberge, P. \& Lessard, L. (2009). Plan d'action québécois en santé mentale : contextes de mise en oeuvre et éléments d'impact sur l'organisation des services de première ligne et les modes de collaboration. Santé mentale au Québec, 34(1), 35-53. https://doi.org/10.7202/029758ar
Résumé de l'article

Depuis 2005, le Plan d'action en santé mentale oriente le développement et l'organisation des services en santé mentale au Québec. En conjonction avec d'autres réformes modifiant l'économie générale du système de santé, il vise en particulier à favoriser une réponse adéquate aux troubles mentaux courants. Cette préoccupation appelle une transformation de l'offre de services en première ligne. Une analyse de différents contextes dans lesquels opèrent ces changements permet une réflexion sur les principaux facteurs susceptibles d'influencer l'actualisation de certaines propositions du Plan d'action et sur l'évolution des modes de collaboration, un pré requis à la mise en place des réseaux locaux de services. 


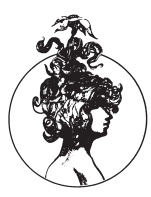

\title{
Plan d'action québécois en santé mentale : contextes de mise en œuvre et éléments d'impact sur l'organisation des services de première ligne et les modes de collaboration
}

\author{
Catherine Vallée* \\ Léo-Roch Poirier** \\ Denise Aubé ${ }^{\star * *}$ \\ Louise Fournier**** \\ Malijaï Caulet ${ }^{\star * * * *}$ \\ Pasquale Roberge ${ }^{\star \star \star \star * *}$ \\ Lily Lessard ${ }^{* * * * * *}$
}

Depuis 2005, le Plan d'action en santé mentale oriente le développement et l'organisation des services en santé mentale au Québec. En conjonction avec d'autres réformes modifiant l'économie générale du système de santé, il vise en particulier à favoriser une réponse adéquate aux troubles mentaux courants. Cette préoccupation appelle une transformation de l'offre de services en première ligne. Une analyse de différents contextes dans lesquels opèrent ces changements permet une réflexion sur les principaux facteurs susceptibles d'influencer l'actualisation de certaines propositions du Plan d'action et sur l'évolution des modes de collaboration, un pré requis à la mise en place des réseaux locaux de services.

$\mathbf{U}$

n des principaux objectifs du Plan d'action en santé mentale (PASM - ministère de la Santé et des Services sociaux, 2005) est de consolider les services de première ligne en vue d'offrir une réponse plus adéquate aux besoins des personnes atteintes de troubles mentaux.

\footnotetext{
* Stagiaire postdoctorale, Institut national de santé publique du Québec.

** Agent de recherche, Institut national de santé publique du Québec.

*** Médecin-conseil, Institut national de santé publique du Québec.

**** Chercheure, Centre de recherche du CHUM (CRCHUM).

***** Agente de recherche, Centre de recherche, Hôpital Douglas.

****** Chercheure, Centre de recherche du CHUM (CRCHUM).

******** Professeur adjointe, Université du Québec à Rimouski.
} 
Cette consolidation s'inscrit dans un contexte de réformes visant une meilleure intégration des différents niveaux de services, notamment par la création des Centres de santé et de services sociaux (CSSS) et l'élaboration de projets cliniques.

À cette fin, le PASM préconise plusieurs mesures dont: 1) la mise en œuvre d'équipes multidisciplinaires de première ligne dans tous les CSSS ; 2) l'ajout de professionnels répondants dont le travail consiste à soutenir cliniquement la pratique des intervenants de première ligne; 3) la mise en œuvre de guichets d'accès centralisés en première ligne, afin de mieux aiguiller les requêtes de services destinées autant en première ligne que vers les services spécialisés. La première ligne devient donc la voie d'accès privilégiée à l'ensemble des services de santé mentale tout en étant un lieu de traitement et d'intégration des soins offerts à un individu (MSSS, 2005), comme le préconisent plusieurs auteurs (Levesque et al., 2007; Starfield, 1998).

Les observations présentées dans cet article sont tirées d'un des volets du projet Dialogue ${ }^{1}$, qui a pour objectif général d'évaluer l'impact des transformations dans l'offre de services de première ligne recommandées par le PASM. L'objectif spécifique du présent volet est d'identifier les éléments contextuels qui affectent la mise en œuvre des orientations du PASM. Il se déroule sur 15 territoires de $\mathrm{CSSS}^{2}$, situés dans huit régions du Québec.

\section{Méthodologie}

Le choix des territoires a été effectué de manière à refléter la plus grande diversité possible des conditions de mise en œuvre du PASM. Les facteurs considérés étaient, entre autres, d'ordre géographique, démographique, historique (dans la prise en charge de la santé mentale) ou structurel (par exemple, la disponibilité de ressources spécialisées). La classification du Collège des médecins du Québec distinguant régions universitaires, périphériques, intermédiaires et éloignées a permis une première approximation des caractéristiques géographiques et structurelles. Deux Agences régionales de santé et des services sociaux sélectionnées dans chaque catégorie en fonction de leur degré de contraste par rapport à l'ensemble des facteurs, ont par la suite été invitées à participer à l'étude. Ces dernières ont accepté d'emblée et ont identifié des répondants régionaux avec lesquels nous avons ciblé des territoires locaux.

Les données utilisées proviennent d'entrevues semi-structurées individuelles et de groupes auprès d'informateurs clés de chaque territoire et de documents qui permettent de mieux comprendre certains 
éléments de mise en œuvre, telle la description de structures de concertation locale, d'un guichet d'accès ou de la programmation.

La première vague de collecte de données en 2006 a été réalisée par le biais de groupes de discussion d'une durée de deux heures, réalisés sur chacun des quinze territoires. La composition des groupes de discussion vise à représenter la diversité des partenaires qui compose le réseau local de services en santé mentale, et est établie par les répondants locaux. Les enregistrements, les verbatim et les notes de recherche ont permis une synthèse qui a été envoyée aux participants pour fins de validation. La première année du projet a permis de dégager des facteurs historiques susceptibles d'influencer l'organisation actuelle des services au sein des CSSS et des réseaux locaux de services, tout en s'attardant au degré d'intégration des ressources. Parmi les aspects documentés figurent: l'évolution du nombre, du type et de l'organisation des ressources en santé mentale, la composition, les modalités d'encadrement et le fonctionnement de l'équipe de santé mentale du CSSS, l'implantation de mesures spécifiques définies par le PASM, telles que le guichet d'accès et le rôle de répondant. Les éléments qui facilitent ou freinent la mise en œuvre de ce plan ont aussi retenu notre attention.

Lors de la collecte réalisée l'année suivante (2007-2008), les groupes de discussion ont été enrichis pour mieux refléter les réalités des réseaux locaux de services. Des entrevues individuelles ont été réalisées auprès des répondants locaux et régionaux afin de mieux circonscrire la mise en œuvre des équipes de santé, du guichet d'accès, du rôle de répondant, l'évolution du projet clinique ou les enjeux locaux d'implantation. De même, des entrevues individuelles ont été réalisées avec des omnipraticiens pour sonder leurs perspectives. Outre une mise à jour des dimensions déjà documentées, cette collecte s'est intéressée davantage aux rapports de collaboration qui existent au sein des CSSS et dans les réseaux locaux de services. Les synthèses réalisées ont fait l'objet d'un accord inter-juge par deux membres de l'équipe assignés au territoire; une validation croisée a aussi été effectuée entre les différentes sources de données. Cet article présente une analyse préliminaire qui repose sur l'ensemble de l'information disponible en date de mai 2008. Les participants occupent principalement des postes de gestion. Le tableau 1 témoigne de la répartition des participants. 


\section{Tableau 1}

\section{Distribution des informateurs clés selon les collectes de données}

\begin{tabular}{|l|c|c|c|}
\hline & $\begin{array}{l}\text { Nombre de } \\
\text { participants à la } \\
\text { tournée 2006 }\end{array}$ & $\begin{array}{l}\text { Nombre } \\
\text { préliminaire de } \\
\text { participants à la } \\
\text { tournée 2007-2008 }\end{array}$ & $\begin{array}{l}\text { Nombre total de } \\
\text { participants }\end{array}$ \\
\hline $\begin{array}{l}\text { Gestionnaires } \\
1^{\text {re }} \text { ligne } \\
2^{\mathrm{e}} \text { ligne }\end{array}$ & $\begin{array}{c}45(38 \%) \\
11(9 \%)\end{array}$ & $\begin{array}{c}31(29 \%) \\
8(7 \%)\end{array}$ & $\begin{array}{c}58(32 \%) \\
18(10 \%)\end{array}$ \\
\hline $\begin{array}{l}\text { Intervenants } \\
1^{\text {re }} \text { ligne } \\
2^{\mathrm{e}} \text { ligne }\end{array}$ & $\begin{array}{c}23(19 \%) \\
7(6 \%)\end{array}$ & $\begin{array}{c}22(21 \%) \\
5(5 \%)\end{array}$ & $\begin{array}{c}36(20 \%) \\
9(5 \%)\end{array}$ \\
\hline $\begin{array}{l}\text { Représentants } \\
\text { d'organismes } \\
\text { communautaires }\end{array}$ & $7(6 \%)$ & $13(12 \%)$ & $18(10 \%)$ \\
\hline Omnipraticiens & $8(7 \%)$ & $8(7 \%)$ & $14(8 \%)$ \\
\hline Psychiatres & $4(3 \%)$ & $5(5 \%)$ & $9(5 \%)$ \\
\hline $\begin{array}{l}\text { Représentants des } \\
\text { agences }\end{array}$ & $8(7 \%)$ & $5(5 \%)$ & $8(4 \%)$ \\
\hline $\begin{array}{l}\text { Proches et personnes } \\
\text { utilisatrices }\end{array}$ & $0(0 \%)$ & $3(3 \%)$ & $3(2 \%)$ \\
\hline Autres & $4(3 \%)$ & $9(8 \%)$ & $12(7 \%)$ \\
\hline $\begin{array}{l}\text { Représentants de CR } \\
\text { en toxicomanie }\end{array}$ & $1(1 \%)$ & $2(2 \%)$ & $2(1 \%)$ \\
\hline TOTAL & 118 & 107 & 182 \\
\hline
\end{tabular}

Note: Comme plusieurs personnes assument plus d'un rôle, les proportions exprimées dans chaque colonne dépassent $100 \%$. Il est à noter également que la seconde collecte de données était complétée dans huit des quinze territoires au moment de la rédaction de l'article.

\section{Stratégie d'analyse}

Aux fins de l'article, l'analyse cible deux objets: les équipes de santé mentale et leurs caractéristiques; et les dynamiques qui prévalent dans les réseaux locaux de services, notamment la collaboration entre les différents acteurs dans un contexte d'appropriation du PASM.

Pour le premier objet, l'analyse met en contraste différentes modalités d'organisation de services selon leur degré de cohérence avec les principales orientations du PASM. Cinq éléments ont retenu l'attention: la mise en œuvre d'une équipe multidisciplinaire de santé mentale en première ligne, et sa capacité de répondre aux besoins de l'ensemble des clientèles associées à la première ligne; l'accès aux services médicaux, généraux ou spécialisés; l'accès à un mentorat, peu importe sa nature; la mise en œuvre du guichet d'accès, qui permet 
d'accéder à l'ensemble des services du réseau local; et enfin l'étendue de la gamme de services au sein des réseaux locaux. C'est en comparant de façon constante les territoires étudiés en fonction des variables retenues, de manière inductive, que se dégagent les résultats préliminaires sur la mise en œuvre des mesures du PASM.

L'analyse des dynamiques de collaboration s'appuie sur un cadre conceptuel développé à partir d'écrits sur la collaboration interprofessionnelle ou inter-organisationnelle (D'Amour et al., 2005 ; D'Amour et al., 2003a, 2003b ; Fleury et al., 2002 ; Gray, 1985 ; Langlois et al., 2003). Selon ce cadre, la collaboration s'élabore en fonction de trois étapes qui s'influencent mutuellement de façon non linéaire: l'appropriation collective des enjeux, l'élaboration d'une communauté de vues et la structuration du réseau local de services. Ce processus est modulé par les pressions extérieures qui incitent les acteurs du réseau à s'engager dans une démarche collaborative, et aussi par leurs efforts respectifs pour influencer le contexte dans lequel elle s'opère. D'autres éléments participent à ce processus de collaboration dont: 1) une implication continue d'acteurs représentant l'exhaustivité des partenaires concernés;2) une interdépendance; 3) une intensité des liens, incluant des contacts en personne; 4) des meneurs crédibles et compétents : 5) la présence ou le développement de gains perceptibles (se référer à la Figure 1).

Figure 1

\section{Cadre conceptuel : la collaboration au sein du réseau local de services}

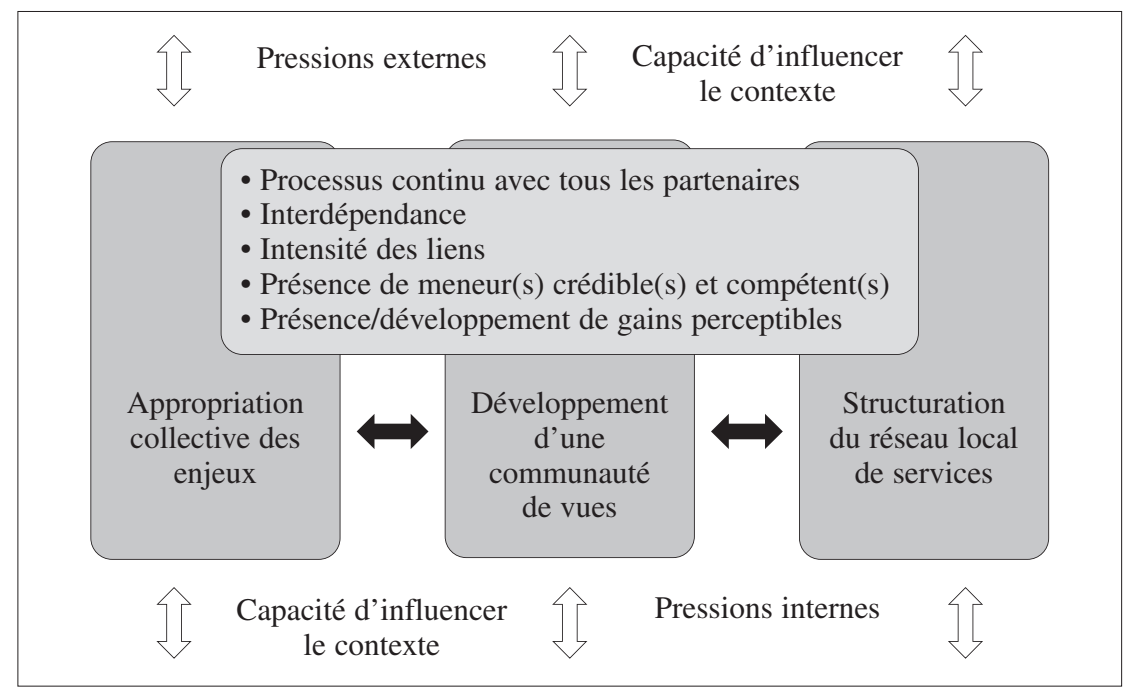




\section{Résultats}

\section{Mise en ouvre du PASM}

À partir des éléments décrits pour établir les comparaisons, les territoires se répartissent en trois groupes. Dans cinq territoires, l'organisation des services semble refléter étroitement plusieurs orientations du PASM. Cinq autres territoires s'en éloignent, alors que les cinq derniers implantent partiellement ces orientations.

\section{Les équipes de santé mentale au sein des CSSS}

Les équipes de santé mentale développées au sein des CSSS se présentent sous diverses configurations qui ne peuvent être expliquées par les données démographiques, le statut socioéconomique de la population, le degré d'implantation de services spécialisés en santé mentale ou les pénuries d'effectifs médicaux, notamment des omnipraticiens. Ainsi, le tableau 2 illustre que pour chaque groupe, le type ou la taille du CSSS ne laisse pas présager du degré d'implantation des mesures du PASM.

Tableau 2

Quelques caractéristiques des CSSS en fonction du degré d'implantation des mesures du PASM

\begin{tabular}{|c|c|c|c|}
\hline & $\begin{array}{l}\text { Implantation plus } \\
\text { soutenue des } \\
\text { mesures du PASM }\end{array}$ & $\begin{array}{c}\text { Implantation } \\
\text { modérée des } \\
\text { mesures du PASM }\end{array}$ & $\begin{array}{c}\text { Implantation } \\
\text { lacunaire des } \\
\text { mesures du PASM }\end{array}$ \\
\hline Type de CSSS & $\begin{array}{l}\text { - } 1 \text { CSSS en région } \\
\text { universitaire } \\
\text { - } 2 \text { CSSS en région } \\
\text { périphérique } \\
\text { - } 2 \text { CSSS en région } \\
\text { éloignée }\end{array}$ & $\begin{array}{l}-2 \text { CSSS en région } \\
\text { universitaire } \\
\text { - } 2 \text { CSSS en région } \\
\text { périphérique } \\
\text { - } 1 \text { CSSS en région } \\
\text { éloignée }\end{array}$ & $\begin{array}{l}\text { - } 2 \text { CSSS en région } \\
\text { universitaire } \\
\text { - } 3 \text { CSSS en région } \\
\text { intermédiaire }\end{array}$ \\
\hline $\begin{array}{l}\text { Population } \\
\text { desservie }\end{array}$ & $\begin{array}{l}\text { - } 4 \text { CSSS : }<60000 \\
\text { habitants } \\
\text { 1 CSSS : }>200000 \\
\text { habitants }\end{array}$ & $\begin{array}{l}\text { - } 4 \text { CSSS : entre } \\
100000 \text { et } 200000 \\
\text { habitants } \\
\text { - } 1 \text { CSSS : > } 200000 \\
\text { habitants }\end{array}$ & $\begin{array}{l}\text { - } 4 \text { CSSS : entre } \\
100000 \text { et } 200000 \\
\text { habitants } \\
\text { 1 CSSS : > } 200000 \\
\text { habitants }\end{array}$ \\
\hline
\end{tabular}

D'autres variables semblent expliquer davantage ces variations. Ainsi, la présence ou l'absence d'une mission hospitalière au sein du CSSS et la localisation intra ou extra territoriale du département de psychiatrie appelé à desservir le territoire influencent la configuration et le fonctionnement des équipes. De même, l'étendue du territoire justifie de ne pas former d'équipe formelle de santé mentale, et de privilégier le 
déploiement des professionnels dédiés à la santé mentale dans les structures existantes que sont l'Accueil-Orientation-Référence (AEO) et les services psychosociaux courants. D'autres milieux considèrent que leur équipe de santé mentale relève d'une deuxième ligne de services et qu'elle soutient les omnipraticiens qui, eux, constituent clairement la première ligne. Plusieurs équipes dites de première ligne sont constituées de professionnels issus de la deuxième ligne qui desservent presque exclusivement une clientèle avec des troubles mentaux graves. Par ailleurs, le suivi intensif dans le milieu ou d'intensité variable demeure peu répandu dans les réseaux locaux à l'étude. En 2006, plusieurs territoires étaient toujours en attente de transfert de ressources ou de fonds de développement. En 2007, on assiste à une injection importante de ressources financières en première ligne contrée par la difficulté à combler les nombreux nouveaux postes.

Les résultats observés dans les territoires où l'organisation des services reflète plus étroitement les recommandations du PASM, s'inscrivent dans une continuité historique. En effet, ces territoires avaient déjà instauré de façon itérative ces changements tout au long de nombreuses années, le PASM ne faisant qu'entériner une vision et des façons de faire déjà bien incarnées. Des fusions successives avaient déjà été réalisées avec une harmonisation satisfaisante des différentes cultures organisationnelles. Ces territoires ont connu une plus grande stabilité des ressources humaines, notamment des gestionnaires. Presque tous ont adopté un mode de fonctionnement hospitalier où les omnipraticiens demeurent les médecins traitants et sont appuyés sur demande par les psychiatres. L'accès à l'expertise du psychiatre demeure facile, malgré les inévitables contraintes. Toutefois, des difficultés persistent, tels que la participation et l'engagement des omnipraticiens ou des contraintes qui appellent des adaptations et des mécanismes de coordination clinique importants.

À l'inverse, les territoires qui s'éloignent des recommandations du PASM se caractérisent par d'importants cloisonnements entre les services de première ligne et les services spécialisés; il arrive même que la deuxième ligne s'oppose ouvertement aux initiatives de la première ligne. L'accès à l'expertise psychiatrique demeure problématique et les omnipraticiens peu engagés. Des changements répétés de gestionnaires, et dans une moindre mesure de professionnels, sont observés. Généralement, ces territoires se confrontent à des réalités complexes où se combinent divers éléments tels la présence de populations plus vulnérables, des cultures organisationnelles peu harmonisées à l'intérieur du CSSS, un historique d'échecs répétés ou de conflits persistants entre partenaires ou encore, un manque chronique d'effectifs. 
Quant aux territoires qui appliquent partiellement les recommandations du PASM, ils se démarquent par un passé plus tumultueux avec les services psychiatriques. On note maintenant une implication où s'exprime un leadership constructif de la part de la deuxième ligne. La hiérarchisation des services gagnerait à être harmonisée, puisque des clientèles de deuxième ligne trouvent une réponse adaptée en première ligne et vice versa. Le tableau 3 regroupe les facteurs susceptibles d'influencer l'implantation des équipes de première ligne.

Tableau 3

\section{Caractéristiques susceptibles d'influencer le degré d'implantation} des équipes de première ligne

\begin{tabular}{|c|c|c|c|}
\hline & $\begin{array}{l}\text { Implantation plus } \\
\text { soutenue des } \\
\text { mesures du PASM }\end{array}$ & $\begin{array}{l}\text { Implantation } \\
\text { modérée des } \\
\text { mesures du PASM }\end{array}$ & $\begin{array}{l}\text { Implantation } \\
\text { lacunaire des } \\
\text { mesures du PASM }\end{array}$ \\
\hline $\begin{array}{l}\text { Considérations } \\
\text { historiques }\end{array}$ & $\begin{array}{l}\text { - Les mesures } \\
\text { s'inscrivent dans } \\
\text { une continuité } \\
\text { historique } \\
\text { - Fusions } \\
\text { organisationnelles } \\
\text { déjà complétées }\end{array}$ & $\begin{array}{l}\text { - Passé souvent } \\
\text { difficile pour les } \\
\text { services spécialisés, } \\
\text { qui désirent par } \\
\text { ailleurs s'affirmer }\end{array}$ & $\begin{array}{l}\text { - Longue histoire de } \\
\text { services et de } \\
\text { gestion non } \\
\text { intégrées }\end{array}$ \\
\hline $\begin{array}{l}\text { Services de } 2^{\mathrm{e}} \\
\text { ligne et } \\
\text { médicaux }\end{array}$ & $\begin{array}{l}\text { - Pour } 2 \text { CSSS, à } \\
\text { l'extérieur du } \\
\text { territoire } \\
\text { - Prise en charge par } \\
\text { les omnipraticiens, } \\
\text { appuyés des } \\
\text { psychiatres } \\
\text { - Expertise } \\
\text { psychiatrique } \\
\text { accessible }\end{array}$ & $\begin{array}{l}\text { - Présence d'un } \\
\text { leadership } \\
\text { constructif de la } 2^{\mathrm{e}} \\
\text { ligne envers la } 1^{\mathrm{e}} \\
\text { - Chevauchement des } \\
\text { lignes de services }\end{array}$ & $\begin{array}{l}\text { - Pour } 2 \text { CSSS, à } \\
\text { l'extérieur du } \\
\text { territoire } \\
\text { - Cloisonnement et } \\
\text { conflits entre la } 1^{\text {re }} \\
\text { et la } 2^{\mathrm{e}} \text { ligne } \\
\text { - Omnipraticiens peu } \\
\text { engagés }\end{array}$ \\
\hline $\begin{array}{l}\text { Mouvements } \\
\text { des } \\
\text { gestionnaires et } \\
\text { du personnel }\end{array}$ & $\begin{array}{l}\text { - Relative stabilité des } \\
\text { gestionnaires et des } \\
\text { intervenants }\end{array}$ & $\begin{array}{l}\text { - Instabilité chez les } \\
\text { gestionnaires }\end{array}$ & $\begin{array}{l}\text { - Instabilité chez les } \\
\text { gestionnaires et les } \\
\text { intervenants }\end{array}$ \\
\hline $\begin{array}{l}\text { Mécanismes de } \\
\text { concertation }\end{array}$ & $\begin{array}{l}\text { - Concertation } \\
\text { présente depuis } \\
\text { plusieurs années, } \\
\text { enrichie par les } \\
\text { projets cliniques }\end{array}$ & $\begin{array}{l}\text { - Volonté d'établir } \\
\text { une concertation } \\
\text { soutenue, } \\
\text { confrontée à des } \\
\text { enjeux } \\
\text { organisationnels }\end{array}$ & $\begin{array}{l}\text { - Négociations } \\
\text { difficiles avec la } \\
\text { deuxième ligne } \\
\text { - Crainte de perdre } \\
\text { des effectifs ou } \\
\text { attente de transferts }\end{array}$ \\
\hline
\end{tabular}




\section{Les mécanismes d'accès}

Le développement de guichets d'accès formels, tel que défini par le PASM, était peu répandu à l'automne 2006. Plusieurs milieux ne priorisaient pas leur mise en œuvre. Certains étaient en attente de ressources humaines nécessaires à leur développement, d'autres cherchaient prioritairement à consolider les équipes et à harmoniser les services afin de ne pas créer une liste d'attente indue.

Dans plusieurs cas, différentes structures existantes ont été mises à profit pour répondre aux fonctions d'un guichet d'accès. Les services de l'AEO des CSSS restent très sollicités. Certains milieux ont tenté d'optimiser l'implication d'intervenants de liaison ou de miser sur des équipes réduites ayant pour mandat d'aiguiller les références et de coordonner les efforts du réseau local de services. Enfin, certains CSSS, situés dans des territoires ruraux où les omnipraticiens sont fortement impliqués, privilégient un guichet unique pour toutes les clientèles.

Depuis 2007-2008, la mise en œuvre du guichet d'accès est désormais au cœur des préoccupations d'une majorité de CSSS. Les activités et les processus des guichets existants sont mieux définis. Plusieurs reconnaissent que le guichet d'accès peut constituer un carrefour pour l'ensemble des services du réseau local et contribuer positivement à l'intégration des services au sein de ce réseau. Mais lorsque la deuxième ligne collabore peu, le champ d'action du guichet d'accès se restreint aux programmes particuliers du CSSS. Si le guichet facilite l'accès à l'évaluation et l'orientation des références, cette mesure s'avère insuffisante pour assurer l'accès au traitement, puisque d'importantes listes d'attente subsistent dans bon nombre de territoires.

\section{Le mentorat au sein des réseaux locaux de services}

Au départ, le PASM insistait sur la désignation de psychiatres répondants, qui devaient devenir les interlocuteurs privilégiés des intervenants de première ligne (MSSS, 2005). Toutefois, cette mesure a soulevé l'opposition de l'Association des médecins psychiatres du Québec. En 2006, peu de psychiatres acceptent cette désignation, même si certains d'entre eux assument des rôles de consultants, de formateurs ou une fonction-conseil.

En juin 2007, le concept de répondant fut élargi pour inclure une variété de professionnels (Delorme, 2007), reflétant ainsi une réalité présente sur le terrain. Par exemple, des omnipraticiens dont la pratique est fortement ancrée en santé mentale jouent déjà un rôle-conseil auprès de leurs collègues. D'autres professionnels sont formellement identifiés 
afin de soutenir des intervenants de première ligne œuvrant au CSSS ou dans des organismes communautaires. Ces professionnels, issus d'horizons divers et souvent sur la base de leur crédibilité, prodiguent non seulement leurs conseils, mais offrent du mentorat via des formations, des discussions de cas ou des suivis conjoints.

Ces fonctions de mentorat sont reconnues au sein des réseaux locaux comme des modalités qui permettent d'accroître l'expertise et les compétences des intervenants de première ligne. Plus spécifiquement, lorsqu'un psychiatre ou un omnipraticien accepte d'assumer formellement des fonctions de mentorat, l'intérêt et la participation des omnipraticiens au sein des structures cliniques ainsi que la prise en charge des clientèles vulnérables et orphelines augmentent.

\section{La collaboration au sein des réseaux locaux de services}

Appropriation collective des enjeux

Une participation continue des partenaires concernés s'avère déterminante pour l'appropriation collective des enjeux. Or, si les services de deuxième ligne et les équipes de première ligne sont souvent en contact étroit, les organismes communautaires sont surtout sollicités pour des dossiers spécifiques tels la prévention du suicide ou l'intégration sociale. Peu d'efforts sont déployés pour collaborer avec les psychologues du secteur privé. Les omnipraticiens déplorent le manque de reconnaissance à leur égard concernant leur expertise ou les caractéristiques particulières de leur pratique. Isolés, ils ont peu d'occasions de rencontrer d'autres acteurs du réseau local de services et sont souvent informés a posteriori des décisions. Les omnipraticiens attendent des effets perceptibles sur les services pour s'impliquer. En l'absence de résultats tangibles ou probants, les efforts des leaders locaux restent vains. Mais plusieurs omnipraticiens témoignent de difficultés à concilier la culture médicale avec la culture psychosociale des équipes de santé mentale. Ils identifient pourtant des expériences probantes où ils ont pu mieux s'arrimer avec le CSSS (par exemple, les services aux personnes en perte d'autonomie).

Les territoires où sont déjà implantés plusieurs éléments du PASM se démarquent par une forte reconnaissance de la légitimité, de l'expertise et de l'interdépendance des différents acteurs du réseau local, dont les omnipraticiens. Une forte connectivité est observée autant à un niveau clinique et organisationnel, qu'entre les cliniciens et les gestionnaires. Des leaders crédibles susceptibles de rallier les partenaires sont présents, dont encore une fois, les omnipraticiens. Toutefois, l'engagement des psychiatres à épauler la première ligne et à développer des 
pratiques de soins partagés demeure déterminant. Les omnipraticiens ont tendance à vouloir surtout utiliser les services psychiatriques; de leur côté, plusieurs psychiatres reconnaissent et confirment la légitimité et la pertinence des équipes de santé mentale de première ligne, encourageant ainsi leurs confrères à les utiliser davantage.

Les réseaux locaux qui s'éloignent des mesures préconisées par le PASM se caractérisent par des conflits entre les équipes de santé mentale de première et de deuxième ligne. Les psychiatres s'opposent ouvertement aux réformes en cours. Les omnipraticiens sont parfois hésitants, le plus souvent absents. Un pôle de collaboration migre lentement vers les équipes de première ligne pour des objets de collaboration demeurant ponctuels et spécifiques. Les occasions de se côtoyer en dehors de l'organisation demeurent limitées tant pour les gestionnaires que pour les cliniciens.

Pour les territoires qui ont partiellement implanté les mesures du PASM, les discussions sur les mécanismes de collaboration inter-organisationnelle au sein du réseau local de services se limitent principalement aux gestionnaires. La connectivité se développe soit entre gestionnaires, soit entre cliniciens. Les intervenants participent peu à l'identification et à l'appropriation des enjeux. Les leaders qui portent la vision du PASM ne font pas l'unanimité. La figure 2 (page suivante) illustre les variations observées dans l'appropriation collective des enjeux selon le degré d'implantation du PASM.

\section{Le développement d'une communauté de vues}

Les données de 2007-2008 rendent compte de l'énergie déployée dans plusieurs territoires pour développer une communauté de vues. Des tractations visent à mieux préciser la hiérarchisation des services, à circonscrire les niveaux de services attendus et les clientèles cibles des différents services. Des discussions portent sur l'interface entre le suivi d'intensité variable et le suivi intensif dans le milieu, l'arrimage entre la première et la deuxième ligne dans le traitement des troubles de la personnalité limite ou des troubles mentaux graves, la mise en place du guichet d'accès, et le déploiement de l'expertise sur le territoire. Cependant, le continuum de services destinés aux personnes avec des troubles mentaux courants retient moins l'attention.

Un partage de l'information de nature administrative susceptible de nourrir la réflexion sur ces divers enjeux génère un contexte plus propice au développement d'une communauté de vues. Or, lorsqu'il existe, le partage d'informations s'effectue d'abord entre établissements et, ponctuellement, avec les organismes communautaires. Des 


\section{Figure 2}

\section{L'appropriation collective des enjeux, selon le degré d'implantation du PASM}

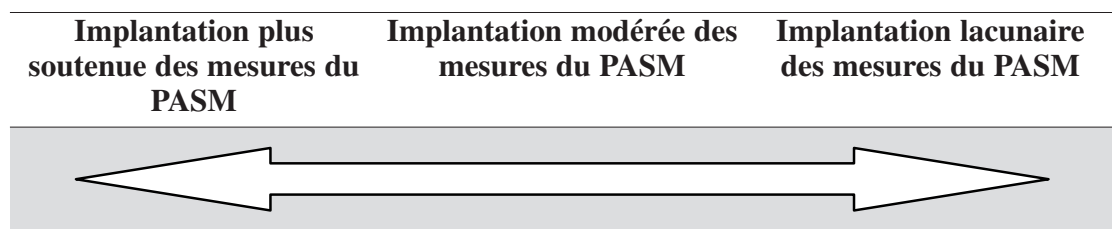

La collaboration existe autour d'une variété d'enjeux
La collaboration existe, mais sur des objets limités et spécifiques

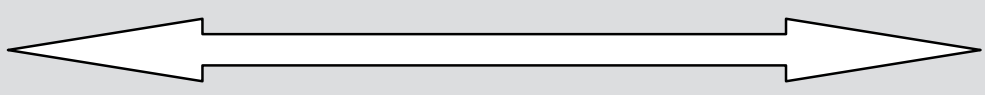

Haute connectivité aux niveaux clinique et organisationnel, autant vertical qu'horizontal

Haute connectivité entre les Peu de connectivité gestionnaires et/ou entre les cliniciens

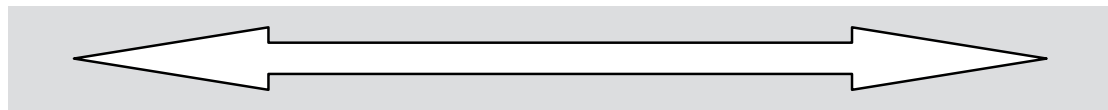

Forte reconnaissance d'interdépendance
Développement d'une interdépendance entre gestionnaires
Tensions marquées entre les services spécialisés et la première ligne

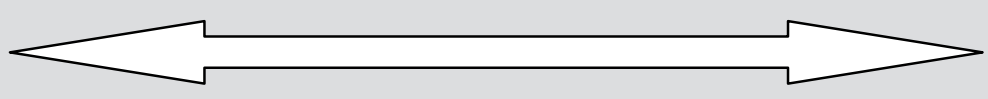

Des leaders crédibles, capables de rassembler le réseau local de services, dont souvent un omnipraticien ; reconnaissance de l'expertise de toutes les parties
Migration du leadership des services spécialisés vers les services de première ligne discrédité par certains acteurs

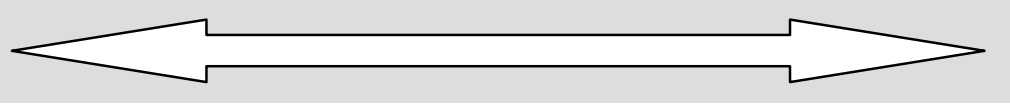

Travail étroit entre psychiatres et omnipraticiens ; les psychiatres jettent des passerelles vers les services de première ligne
Opposition souvent face aux mesures du PASM manifeste des psychiatres 
partenaires importants, tels les Agences, les omnipraticiens, les organismes communautaires ou les services de deuxième ligne, sont souvent absents des délibérations.

Même dans les réseaux locaux plus cohésifs, l'interdépendance n'est pas pour autant reconnue. Néanmoins, plusieurs éléments de la hiérarchisation des services font consensus. Dans les territoires où la collaboration est fragile, l'ouverture de nouveaux chantiers demeure circonscrite. Les travaux se limitent à l'harmonisation des services entre points de services ou entre programmes du CSSS. L'information est rarement partagée et les dialogues sont prudents. Les territoires qui consolident leurs efforts de collaboration élargissent le cadre de discussion. Toutefois, l'équipe de première ligne cherche souvent à se définir d'abord avant d'impliquer ses partenaires. La Figure 3 (page suivante) présente une synthèse des observations recueillies sur le développement d'une communauté de vues.

\section{La structuration des réseaux locaux de services}

La mise en œuvre du guichet d'accès et l'identification de professionnels répondants contribuent à structurer les réseaux locaux de services. Dans les territoires qui intègrent davantage les orientations du PASM, le mentorat s'exerce de diverses façons: il repose sur plusieurs individus et s'adresse tant aux intervenants des programmes généraux qu'aux acteurs du réseau local de services. Les mécanismes de liaison sont bien établis et maintiennent la cohérence des activités du réseau. Il existe un point d'entrée en première ligne, même s'il ne s'agit pas toujours d'un guichet d'accès au sens du PASM. Les réseaux locaux ont parfois développé des protocoles cliniques de soins ou des outils d'évaluation communs. Et même dans ce contexte, des pratiques en silo persistent.

Les territoires ayant implanté partiellement les mesures du PASM sont encore à définir le déploiement de l'expertise et du mentorat. Si le guichet d'accès est en place, il demeure méconnu ou sous-exploité. Des intervenants y assument des fonctions de liaison et favorisent le développement des collaborations. Des ententes de services existent principalement entre le CSSS et les organismes communautaires.

Les territoires qui ont de la difficulté à implanter les mesures du PASM témoignent d'une faible connectivité entre les partenaires. Le mentorat s'appuie généralement sur l'implication d'un seul individu, le plus souvent un omnipraticien ou un psychiatre alors que d'autres professionnels soutiennent les intervenants des programmes généraux. Le guichet d'accès est en voie d'élaboration; entre-temps, les intervenants de liaison jouent un rôle névralgique au sein du réseau local de services. 
Figure 3

Le développement d'une communauté de vues, selon le degré
d'implantation du PASM

\begin{tabular}{ccc}
\hline $\begin{array}{c}\text { Implantation plus } \\
\text { soutenue des mesures du } \\
\text { PASM }\end{array}$ & $\begin{array}{c}\text { Implantation modérée des } \\
\text { mesures du PASM }\end{array}$ & $\begin{array}{c}\text { Implantation lacunaire } \\
\text { des mesures du PASM }\end{array}$ \\
\hline
\end{tabular}

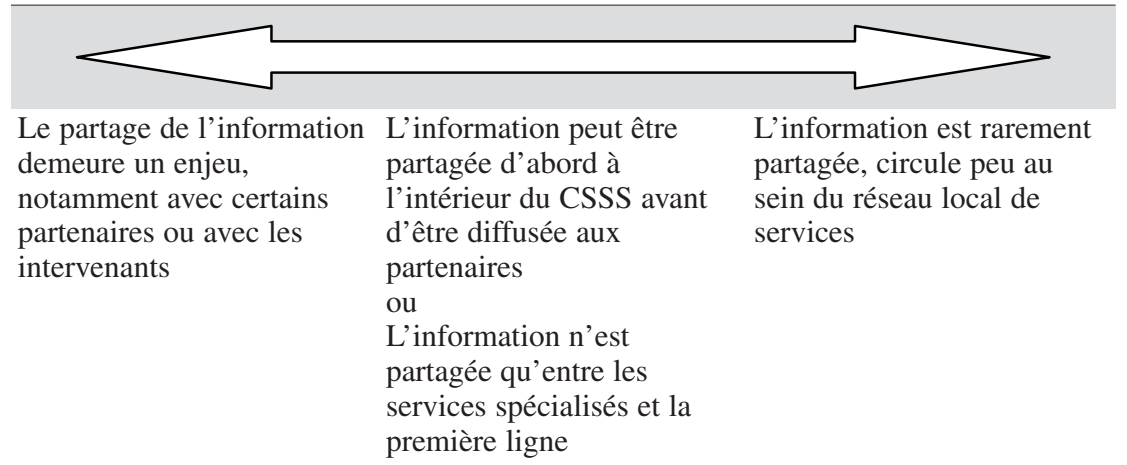

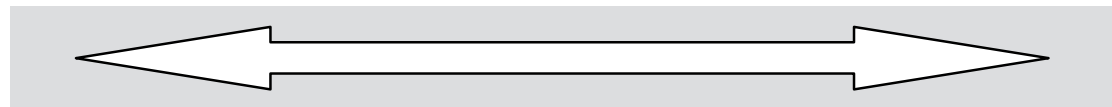

Si les thèmes des discussions sont davantage ouverts, la reconnaissance de l'interdépendance des acteurs du RLS est moins forte
Les thèmes des discussions Les thèmes des discussions tendent à s'élargir se limitent à des sujets bien circonscrits, de peu d'envergure

L'information est rarement partagée, circule peu au sein du réseau local de . 
Le concept d'intervenant-pivot, mis de l'avant par le PASM pour coordonner les services requis par les clientèles vulnérables (MSSS, 2005), est peu abordé par les informateurs. Ceci surprend peu dans un contexte où les partenaires tentent de définir et de s'approprier une hiérarchisation des services qui sied à leur contexte. La Figure 4 reprend les éléments touchant la structuration du réseau local de services.

Figure 4

\section{La structuration des réseaux locaux de services, selon le degré d'implantation du PASM}

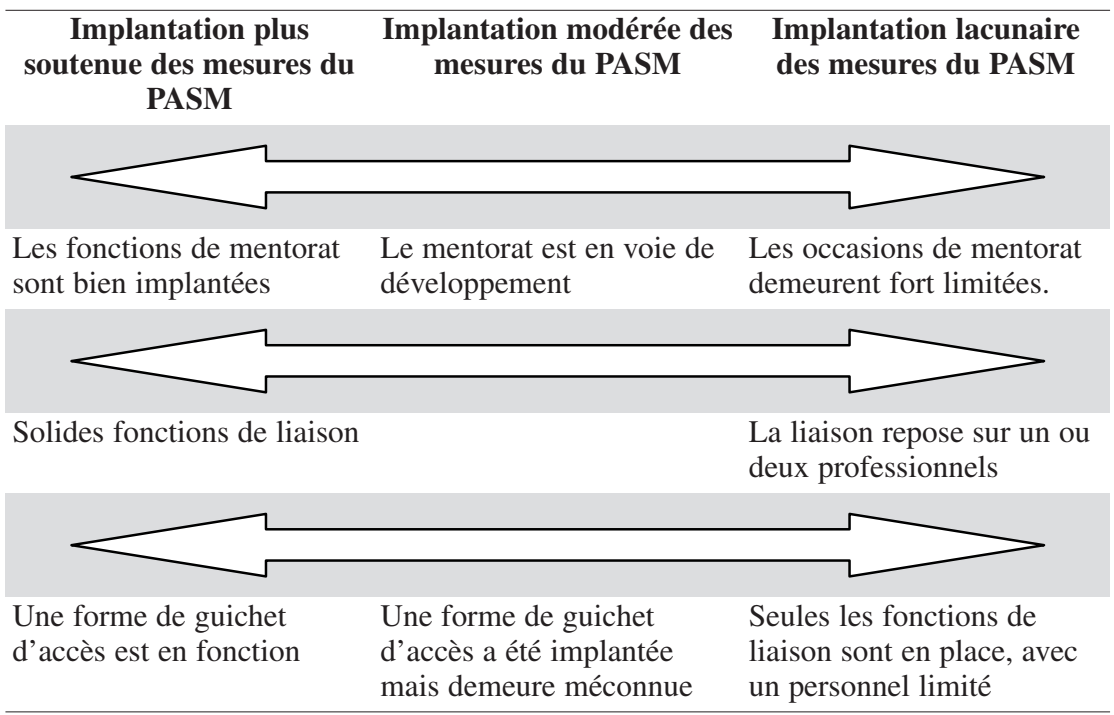

\section{Perspectives pour l'optimisation des services}

Le PASM reconnaît que plusieurs principes sont incontournables à une pratique de soins en collaboration (MSSS, 2005). Nos travaux distinguent des conditions transversales, pouvant affecter chaque étape, et des conditions spécifiques à un moment du développement des rapports de collaboration. Si la reconnaissance d'une interdépendance des partenaires, un leadership éclairé et un engagement continu des partenaires doivent exister tout au long du processus, la définition des rôles et des responsabilités des partenaires prend forme à l'étape du développement d'une communauté de vues et se formalise lors de la structuration, souvent dans le cadre d'ententes de services. Bien que ces ententes soient privilégiées dans le PASM (MSSS, 2005), la structuration du guichet d'accès, l'élaboration des projets cliniques et 
l'appropriation de la hiérarchisation des services contribuent de façon significative à la structuration des réseaux locaux de services.

En particulier, l'élaboration du projet clinique constitue une occasion privilégiée de décloisonner les services. Son actualisation génère des résultats positifs sur l'intégration des structures et des approches (Rousseau et al., 2007). Par ailleurs, des auteurs questionnent la multiplication potentielle de réseaux organisés autour de clientèles spécifiques, craignant la désintégration éventuelle du système (Pineault, 2001) ou l'exclusion des personnes aux prises avec des problématiques complexes, particulièrement si des problèmes sociaux se greffent aux troubles mentaux (Langlois et al., 2003). Ultimement, Pineault (2001) juge plus pertinent d'organiser les services de première ligne en fonction des besoins populationnels et d'assurer des corridors de services fluides et fonctionnels vers les services spécialisés.

\section{Conclusion}

Le PASM s'appuie sur une lecture populationnelle, prenant pour assises les services de première ligne. Cette lecture offre de puissants leviers pour introduire des changements déjà amorcés dans la plupart des réseaux locaux, dont le développement de pratiques en collaboration. La continuité des contacts formels et informels entre partenaires favorise la confiance, la reconnaissance des expertises et l'interdépendance (Cazale et al., 2007). Ces contacts doivent s'opérer à l'intérieur et entre tous les niveaux de décisions afin d'augmenter l'adhésion des parties. Toutefois, les initiatives de collaboration doivent être formalisées, afin qu'elles puissent jouir d'une certaine pérennité (Fleury et al., 2007) sans pour autant désinvestir le maintien de liens personnalisés. Enfin, les réseaux locaux ont avantage à identifier des meneurs crédibles et susceptibles de rallier les partenaires hésitants (Fleury et al., 2007; Langlois et al., 2003; Longest, 1998; Pointer et al., 1995), notamment les omnipraticiens sur lesquels repose la première ligne. De plus, il faudra innover afin de développer des pratiques collaboratives qui puissent concilier les impératifs des établissements et la logique entrepreneuriale de la pratique médicale de première ligne.

\section{Notes}

1. Subventionné par la Fondation canadienne de recherche sur les services de santé, le Fonds de recherche en santé du Québec, l'Institut national de santé publique du Québec, le ministère de la Santé et des Services sociaux du Québec et le Groupe inter universitaire de recherche sur les urgences. Certains résultats préliminaires encore inédits sont disponibles sur le site www.inspq.qc.ca/dialogue. Le projet a été approuvé par les 
comités d'éthique de tous établissements concernés soit directement, soit par délégation auprès d'un autre comité d'éthique.

2. L'étude se déroule aussi dans les régions du nord du Québec mais, compte tenu des enjeux particuliers qui président à l'organisation des services de santé de ces régions, nous n’y ferons pas référence ici.

\section{Références}

Cazale, L., Touati, N., Fleury, M.-J., 2007, La mise en œuvre des réseaux intégrés : expérimentations et évaluations en Montérégie, in Fleury, M.-J. Tremblay, M., Nguyen, H., Bordeleau, L., éds., Le système sociosanitaire au Québec: gouvernance, régulation et participation, Gaëtan Morin, Montréal, 195-217.

D'amour, D., Ferrada-Videla, M., San Martin Rodriguez, L., Beaulieu, M.-D., 2005, The conceptual basis for interprofessional collaboration: core concepts and theoretical frameworks, Journal of Interprofessional Care, 1, Suppl 1, 116-131.

D’amour, D., Goulet, L., Pineault, R., Labadie, J.-F., Remondin, M., 2003a, Étude comparée de la collaboration inter-organisationnelle et de ses effets : le cas des services en périnatalité, Groupe de recherche interdisciplinaire en santé (GRIS) et Centre Ferasi, Montréal.

D’amour, D., Goulet, L., Pineault, R., Labadie, J.-F., Remondin, M., 2003b, Étude comparée de la collaboration inter-organisationnelle et de ses effets: le cas des services en périnatalité, Fondation canadienne de recherche sur les services de santé, Ottawa.

Delorme, A., 2007, Sharing competencies, sharing responsibilities: the Quebec action plan on mental health, présentation effectuée lors de 8th National conference on collaborative mental health care, saisie le 2 juin 2008, http://www.shared-care.ca/qc_presentation.shtml

Fleury, M.-J., Grenier, G., OuAdahi, Y., 2007, Les réseaux intégrés de services: notion, modélisations et mise en œuvre, in Fleury, M.-J. Tremblay, M., Nguyen, H., Bordeleau, L., éds., Le système sociosanitaire au Québec: gouvernance, régulation et participation, Gaëtan Morin, Montréal, 159-178.

Fleury, M.-J., Mercier, C., Denis, J. L., 2002, Regional planning implementation and its impact on integration of a mental health care network, International Journal of Health Planning and Management, 17, 4, 315332.

GRAY, B., 1985, Conditions facilitating interorganisational collaboration, Human Relations, 38, 10, 911-936. 
Langlois, A.-M., St-Pierre, M., BÉGin, C., 2003, Les réseaux de services intégrés: possibilités, limites et enjeux, in Lemieux, V., Bergeron, P., Bégin, C., Bélanger, G., éds., Le système de santé au Québec: organisations, acteurs et enjeux, nouvelle édition, Presses de l'Université Laval, Québec, 145-174.

Levesque, J.-F., Roberge, D., Pineault, R., 2007, La première ligne de soins : un témoin distant des réformes institutionnelles et hospitalières au Québec ?, in Fleury, M.-J. Tremblay, M., Nguyen, H., Bordeleau, L., éds., Le système sociosanitaire au Québec: gouvernance, régulation et participation, Gaëtan Morin, Montréal, 63-78.

LONGEST, B. B., 1998, Managerial competence at senior levels of integrated delivery systems, Journal of Healthcare Management, 43, 2, 115-133.

Ministère de la Santé et Des Services sociaux, 2005, Plan d'action en santé mentale 2005-2010 - La force des liens, Ministère de la Santé et des Services sociaux du Québec.

Pineault, R., 2001, Les propositions de la Commission Clair sont-elles prometteuses? Une analyse de leur cohérence interne et externe, Ruptures Revue transdisciplinaire en santé, 8, 1, 98-105.

Pointer, D. D., Alexander, J. A., Zuckerman, H. S., 1995, Loosening the Gordian knot of governance in integrated health care delivery systems, Frontiers in Health Service Management, 11, 3, 3-37.

Rousseau, L., CaZale, L., Fleury, M.-J., 2007, Réforme ou évolution? Enjeux et opinions sur la régulation et la transformation, in Fleury, M.-J. Tremblay, M., Nguyen, H., Bordeleau, L., éds., Le système sociosanitaire au Québec: gouvernance, régulation et participation, Gaëtan Morin, Montréal, 23-37.

Starfield, B., 1998, Primary Care: Balancing Health Needs, Services and Technology, Oxford University Press, New York.

\section{ABSTRACT}

\section{Mental Health Action Plan: Contextual analysis and elements impacting on organization of primary care services and collaborative issues}

Since 2005, the Mental Health Action Plan maps out development and reorganization of mental health services in Québec. With concurrent reforms affecting the overall layout of the health care system, the Action Plan especially seeks to improve the management of common mental disorders. This particular concern calls for transformations at the primary care level. Contextual analysis of contrasting settings allows the 
identification of the main determinants in this actual process of change and in the ways collaborative issues are addressed.

\section{RESUMEN}

Plan de acción quebequense en salud mental: contextos de la implementación y elementos de impacto en la organización de los servicios de primer nivel y las formas de colaboración

Desde 2005, el Plan de Acción de Salud Mental orienta el desarrollo y la organización de los servicios de salud mental en Quebec. Junto con otras reformas que modifican la economía general del sistema de salud, el plan busca en particular favorecer una respuesta adecuada a los trastornos mentales comunes. Esta preocupación implica una transformación de la oferta de servicios de primer nivel. Un análisis de diferentes contextos en los que operan estos cambios permite una reflexión acerca de los principales factores susceptibles de influir en la actualización de ciertas proposiciones del Plan de Acción y en la evolución de las formas de colaboración, un prerrequisito para la puesta en marcha de las redes locales de servicios.

\section{RESUMO}

Plano de ação quebequense em saúde mental: contextos de aplicação e elementos de impacto na organização dos serviços primários e os modos de colaboração

Desde 2005, o Plano de Ação em Saúde Mental orienta o desenvolvimento e a organização dos serviços em saúde mental no Quebec. Em conjunto com outras reformas que modificam a economia geral do sistema de saúde, ele visa principalmente favorecer uma resposta adequada aos transtornos mentais correntes. Esta preocupação demanda uma transformação da oferta de serviços primários. Uma análise de diferentes contextos nos quais operam estas mudanças permite uma reflexão sobre os principais fatores susceptíveis de influenciar a atualização de algumas propostas do Plano de Ação e sobre a evolução dos modos de colaboração, um pré-requisito para a criação das redes locais de serviços. 